\title{
Influence of the Barotropic Mean Flow on the Width and the Structure of the Atlantic Equatorial Deep Jets
}

\author{
Martin Claus, Richard J. Greatbatch, And Peter Brandt \\ GEOMAR Helmholtz Centre for Ocean Research Kiel, Kiel, Germany
}

(Manuscript received 20 March 2014, in final form 17 June 2014)

\begin{abstract}
A representation of an equatorial basin mode excited in a shallow-water model for a single high-order baroclinic vertical normal mode is used as a simple model for the equatorial deep jets. The model is linearized about both a state of rest and a barotropic mean flow corresponding to the observed Atlantic Equatorial Intermediate Current System. It was found that the eastward mean flow associated with the North and South Intermediate Counter Currents (NICC and SICC, respectively) effectively shields the equator from off-equatorial Rossby waves. The westward propagation of these waves is blocked, and focusing on the equator due to beta dispersion is prevented. This leads to less energetic jets along the equator. On the other hand, the westward barotropic mean flow along the equator reduces the gradient of absolute vorticity and hence widens the cross-equatorial structure of the basin mode. Increasing lateral viscosity predominantly affects the width of the basin modes' Kelvin wave component in the presence of the mean flow, while the Rossby wave is confined by the flanking NICC and SICC. Independent of the presence of the mean flow, the application of sufficient lateral mixing also hinders the focusing of off-equatorial Rossby waves, which is hence an unlikely feature of a low-frequency basin mode in the real ocean.
\end{abstract}

\section{Introduction}

The equatorial deep jets (EDJs) are a common feature of all three equatorial ocean basins. First observational evidence for their existence was found in the Indian Ocean (Luyten and Swallow 1976), followed by the Pacific (Hayes and Milburn 1980; Leetmaa and Spain 1981; Firing 1987) and the Atlantic (Eriksen 1982). They usually take the form of geostrophically balanced, vertically stacked zonal jets that are trapped near the equator and alternate in direction with depth and time. However, coherent signals in the interannual range are only present in the Atlantic basin but not in the Indian Ocean, which is of similar width compared to the Atlantic (Brandt et al. 2011). In the Pacific coherent signals are also observed that are suggested to vary on multidecadal time scales (Johnson et al. 2002). Typically, the Atlantic EDJs as found from observations are characterized by a small vertical scale of 300-700 m, a large zonal scale comparable to the basin width, and a time scale of about $4.5 \mathrm{yr}$.

Corresponding author address: Martin Claus, GEOMAR Helmholtz Centre for Ocean Research Kiel, Düsternbrooker Weg 20, 24105 Kiel, Germany.

E-mail: mclaus@geomar.de
They appear at depths ranging from 200 to $3000 \mathrm{~m}$ with amplitudes exceeding $10 \mathrm{~cm} \mathrm{~s}^{-1}$. Time series of moored zonal velocity observations collected over several years reveal a downward phase propagation of the EDJs (Bunge et al. 2008; Brandt et al. 2011), and both the direction of vertical propagation and the associated time scale are in agreement with estimates by Johnson and Zhang (2003) based on hydrographic station data.

The downward phase propagation is a particularly interesting aspect of the Atlantic EDJs, since according to linear theory this implies upward energy propagation and therefore suggests that they are a mode of variability intrinsic to the ocean with the source of energy at depth. Moreover, distinct variability with a time scale of $4.5 \mathrm{yr}$ can be found in surface parameters such as sea surface temperature (SST), $10 \mathrm{~m}$-winds, rainfall, or zonal surface currents, suggesting that this variability is related to the EDJs (Brandt et al. 2011). A better representation of the EDJs in predictive simulations might therefore improve the climate predictability in the interannual to decadal range in the tropical Atlantic region.

The manifestation of the EDJs as relatively strong zonally coherent jets suggests an impact on the transport of water mass properties and ventilation along the equator. Indeed, Gouriou et al. (2001) found a positive 
correlation of CFC-11 concentration and zonal velocity at $35^{\circ} \mathrm{W}$ on the equator at depths between 1000 and $2500 \mathrm{~m}$ and concluded that the eastward-flowing jets are fed by recently produced North Atlantic Deep Water (NADW) provided by the southward-flowing deep western boundary current (DWBC). Moored observations on the equator at $23^{\circ} \mathrm{W}$ and $300-\mathrm{m}$ depth revealed a pronounced oscillation of oxygen concentration with its period being associated with the EDJs, and a simple idealized advection-diffusion model, driven by an equatorial basin mode, is able to explain this observed oxygen variability (Brandt et al. 2012). Both model and observation show a temporal phase difference between oxygen and zonal velocity of less than quadrature, which indicates a mean eastward transport of oxygen along the equator by the oscillating currents.

The similarity of the EDJs and the gravest equatorial basin mode (Cane and Moore 1981) is often noted, for example, by Johnson and Zhang (2003) and d'Orgeville et al. (2007); however, an exact correspondence is not possible due to the presence of forcing, dissipation, and nonlinear effects. The gravest equatorial basin mode consists of an equatorial Kelvin wave traveling eastward that is reflected at the eastern boundary mostly as a gravest meridional mode long Rossby wave that propagates at a third of the speed of the Kelvin wave. The period $T$ of this oscillation is set by the width of the basin $L$ and the gravity wave speed of the corresponding vertical normal mode $c$ and is 4 times the time it takes the Kelvin wave to cross the basin, that is, $T=4 L / c$. A vertical mode decomposition from observations of zonal velocity at $23^{\circ} \mathrm{W}$ suggests that the energy associated with the EDJs is distributed between vertical modes 10 and 20 with a distinct peak at mode 15 (Brandt et al. 2008). This could explain the apparent vertical propagation of the EDJs with the coexistence of several quasi-resonant high-order baroclinic basin modes.

The properties of the EDJs are in good agreement with linear inviscid theory except for their cross-equatorial width, which is about $50 \%$ larger than one would expect based on their vertical scale (Johnson and Zhang 2003). This discrepancy may have different reasons: (i) Hua et al. (1997) proposed nonlinear effects to broaden the jets; (ii) Greatbatch et al. (2012) argued that turbulent isopycnal mixing of momentum reduces the meridional pressure gradient that is necessary to balance the jets and this requires, in the absence of diapycnal mixing, a broader cross-equatorial width; and (iii) the presence of a barotropic mean flow might affect the width and structure of the EDJs, the subject of this paper. Horizontal shear of the mean flow might reduce the meridional gradient of ambient absolute vorticity at the equator that would result in a larger effective equatorial radius of deformation. Assuming a barotropic zonal mean flow, which depends only on latitude, a necessary condition for an increase in width is a positive curvature of the zonal flow at the equator. In the equatorial Atlantic Ocean, the Equatorial Intermediate Current (EIC), a large verticalscale westward flow, is flanked by eastward jets at $2^{\circ} \mathrm{N} / \mathrm{S}$ called the North and South Intermediate Counter Currents (NICC and SICC, respectively; Richardson and Fratantoni 1999; Gouriou et al. 2001; Ascani et al. 2010; Greatbatch et al. 2012). These currents can create the necessary environment for a reduced meridional gradient of ambient vorticity and hence a widening of the EDJs. Furthermore, the mean flow affects the dispersion properties of the equatorial waves involved in the basin mode. This may change the ray paths of the long Rossby waves radiated at the eastern boundary and hence could significantly impact the cross-equatorial structure.

Here, we excite an equatorial basin mode for a single high-order baroclinic mode in a shallow-water model linearized about a mean state to mimic the EDJs. The model is forced by the oscillating stress of given frequency in the zonal momentum equation, and the isopycnal mixing of momentum is parameterized by Laplacian diffusion in the momentum equations. The sensitivity of the model response to the presence of a mean flow, the magnitude of the eddy viscosity, and the region of applied forcing is analyzed. Additionally, a ray-tracing model, following Schopf et al. (1981; see also Chang and Philander 1989) is used to gain more qualitative insight on the effect of the mean flow on the dynamics of the long equatorial Rossby waves involved in the equatorial basin mode. Actually, in this work, the ray tracing is based on the local dispersion relation of midlatitude Rossby waves and, strictly speaking, cannot be applied to the equatorial band. However, because of the high vertical mode and small equatorial radius of deformation $R_{E} \approx 0.78^{\circ}$, it still gives plausible results in regions close to the equator.

The subsequent section of this paper will cover a description of the employed models and the method used to produce a mean state. In section 3 , the results of the ray-tracing model and the shallow-water model are presented. Discussion and summary are provided in section 4 .

\section{Models and mean flow}

\section{a. Shallow-water model}

We use a shallow-water model of a single high-order baroclinic mode, linearized about a barotropic mean flow, for which the governing equations in spherical coordinates are given by 


$$
\begin{gathered}
\frac{\partial u}{\partial t}-(f+Z) v-\zeta V=-\frac{1}{a \cos \theta} \frac{\partial g \eta+E}{\partial \lambda}+X+M^{u} \\
\frac{\partial v}{\partial t}+(f+Z) u+\zeta U=-\frac{1}{a} \frac{\partial g \eta+E}{\partial \theta}+M^{v}, \quad \text { and } \\
\frac{\partial \eta}{\partial t}+H \nabla \cdot \mathbf{u}+\nabla \cdot(\mathbf{U} \eta)=0
\end{gathered}
$$

where $\lambda$ is longitude, $\theta$ is latitude, $a$ is the radius of Earth, $f=2 \Omega \sin \theta$ is the Coriolis parameter, $g$ is the gravitational acceleration, $H$ is the equivalent depth of the selected vertical mode, and $Z=\operatorname{curl}(U, V)$ and $\zeta=\operatorname{curl}(u, v)$ are the vorticity of the barotropic mean flow $\mathbf{U}=(U, V)$ and of the baroclinic flow $\mathbf{u}=(u, v)$, respectively. The divergence operator used in Eq. (3) and the curl operator used to compute the vorticities $Z$ and $\zeta$ are given in spherical coordinates by

$$
\begin{aligned}
\nabla \cdot(\gamma, \delta) & =\frac{1}{a \cos \theta}\left[\frac{\partial \gamma}{\partial \lambda}+\frac{\partial(\delta \cos \theta)}{\partial \theta}\right], \quad \text { and } \\
\operatorname{curl}(\gamma, \delta) & =\hat{\mathbf{z}} \cdot \nabla \times(\gamma, \delta)=\frac{1}{a \cos \theta}\left[\frac{\partial \delta}{\partial \lambda}-\frac{\partial(\gamma \cos \theta)}{\partial \theta}\right],
\end{aligned}
$$

respectively, where $\hat{\mathbf{z}}$ is the unit vector in the vertical direction. The term $E$ is the perturbed part of the kinetic energy given by $E=u U+v V$ and $\eta$ corresponds to the isopycnal displacement. It should be noted here that we assumed a flat bottom and the barotropic mean flow to be purely rotational, that is, $\boldsymbol{\nabla} \cdot \mathbf{U}=0$, in deriving Eq. (3). The equation

$$
X=\tau^{x} X_{0} \sin \omega_{f} t
$$

is the forcing applied to the zonal momentum equation that is used to excite a basin mode and to maintain it against dissipation. The forcing $X$ is determined by the dimensional scalar amplitude $\tau^{x}$, the frequency $\omega_{f}$, and the dimensionless spatial pattern $X_{0}$. Here, we define five distinct patterns: "full" corresponds to $X_{0}=1$ all over the domain; "west", "center", and "east" have a magnitude of one only in the western, center, or eastern third of the domain, respectively, and zero elsewhere, are meridionally uniform, and there is a steplike transition from forced to unforced regions; finally, "equator" confines the forcing to the equator, having $X_{0}=\exp \left[-(a \theta)^{2} / 2 R_{E}^{2}\right]$, where $R_{E}=\sqrt{c / \beta}$ is the equatorial radius of deformation. We want to point out that the actual value of $\tau^{x}$ is of no greater importance in this context due to the linearity of the model; hence, any change to $\tau^{x}$ would only scale the model response. The lateral mixing of momentum with eddy viscosity $A$ is parameterized in the momentum Eqs. (1) and (2) by $\left(M^{u}, M^{v}\right)$, given by

$$
\begin{aligned}
& M^{u}=A\left[\nabla^{2} u+\frac{u\left(1-\tan ^{2} \theta\right)}{a^{2}}-\frac{2 \sin \theta}{a^{2} \cos ^{2} \theta} \frac{\partial v}{\partial \lambda}\right], \quad \text { and } \\
& M^{v}=A\left[\nabla^{2} v+\frac{v\left(1-\tan ^{2} \theta\right)}{a^{2}}-\frac{2 \sin \theta}{a^{2} \cos ^{2} \theta} \frac{\partial u}{\partial \lambda}\right],
\end{aligned}
$$

where $\nabla^{2}$ is the Laplacian operator in spherical coordinates given by

$$
\nabla^{2} \gamma=\frac{1}{a^{2} \cos ^{2} \theta} \frac{\partial^{2} \gamma}{\partial \lambda^{2}}+\frac{1}{a^{2} \cos \theta} \frac{\partial}{\partial \theta}\left(\cos \theta \frac{\partial \gamma}{\partial \theta}\right) .
$$

To fulfill the required condition of a divergence-free barotropic mean flow we define $(U, V)$ from a prescribed streamfunction $\Psi$, where

$$
(U, V)=\left(-\frac{\partial \Psi}{\partial \theta}, \frac{\partial \Psi}{\partial \lambda}\right)
$$

The spatial discretization is carried out on a regular Arakawa $\mathrm{C}$ grid following the enstrophy conserving scheme of Sadourny (1975), and an Adams-Bashforth second-level scheme is used for time stepping. The domain is specified to roughly the same size as the equatorial Atlantic. It spans $55^{\circ}$ in longitude and reaches to $10^{\circ} \mathrm{N} / \mathrm{S}$ with a resolution of $0.1^{\circ}$ in both latitude and longitude, which is fine enough to resolve the equatorial radius of deformation of the chosen vertical mode of order $1^{\circ}$.

\section{b. Ray-tracing model}

To gain qualitative understanding on the influence of the barotropic mean flow on the basin mode, we trace ray paths of the long Rossby wave excited at the eastern boundary. For this purpose, we use the local dispersion relation of the free and inviscid form of Eqs. (1)-(3), that is, $X=0$ and $A=0$, assuming quasigeostrophy and making use of the Wentzel-Kramers-Brillouin-Jeffries (WKBJ) approximation, hence,

$$
\omega=U k+V l-\frac{k \tilde{f}_{y}-l \tilde{f}_{x}}{k^{2}+l^{2}-R_{c}^{-2}},
$$

where $\omega$ is the frequency, $k$ is the zonal wavenumber, $l$ is the meridional wavenumber, $\tilde{f}=f+Z$ is the ambient vorticity, and $R_{c}=c / f$ is the radius of deformation for a given gravity wave speed $c$. It should be noted here that Eq. (11) is strictly only valid if $\left(a \theta / R_{E}\right)^{2} \gg 1$. In the case of no barotropic mean flow, the local dispersion relation reduces to the same dispersion relation derived 
TABLE 1. Cruises used to compute the barotropic mean flow.

\begin{tabular}{|c|c|c|c|}
\hline Cruise & Date & Section & Max depth (m) \\
\hline Thalassa & August 1999 & $6^{\circ} \mathrm{S}-6^{\circ} \mathrm{N}, 23^{\circ} \mathrm{W}$ & 6000 \\
\hline Seward Johnson & January 2000 & $6^{\circ} \mathrm{S}-4^{\circ} \mathrm{N}, 23^{\circ} \mathrm{W}$ & 2000 \\
\hline Meteor 47/1 & April 2000 & $5^{\circ} \mathrm{S}-4^{\circ} \mathrm{N}, 23^{\circ} \mathrm{W}$ & 5000 \\
\hline Meteor 55 & October 2002 & $0^{\circ}-10^{\circ} \mathrm{N}, 24^{\circ} \mathrm{W}$ & 650 \\
\hline Meteor 68/1 & May 2006 & $2^{\circ} \mathrm{S}-0.5^{\circ} \mathrm{N}, 23^{\circ} \mathrm{W}$ & 500 \\
\hline Ronald H. Brown & June 2006 & $5^{\circ} \mathrm{S}-13.5^{\circ} \mathrm{N}, 23^{\circ} \mathrm{W}$ & 1500 \\
\hline Ronald H. Brown & June-July 2006 & $5^{\circ} \mathrm{S}-14.5^{\circ} \mathrm{N}, 23^{\circ} \mathrm{W}$ & 1500 \\
\hline Meteor 68/2 & June-July 2006 & $4^{\circ} \mathrm{S}-15.25^{\circ} \mathrm{N}, 23^{\circ} \mathrm{W}$ & 1300 \\
\hline Ronald H. Brown & May 2007 & $4^{\circ}-14.5^{\circ} \mathrm{N}, 23^{\circ} \mathrm{W}$ & 1500 \\
\hline L'Atalante & February-March 2008 & $2^{\circ} \mathrm{S}-14^{\circ} \mathrm{N}, 23^{\circ} \mathrm{W}$ & 400 \\
\hline L'Atalante & March 2008 & $2^{\circ} \mathrm{S}-14^{\circ} \mathrm{N}, 23^{\circ} \mathrm{W}$ & 1300 \\
\hline Maria S. Merian 10/1 & November-December 2008 & $4^{\circ}-14^{\circ} \mathrm{N}, 23^{\circ} \mathrm{W}$ & 1000 \\
\hline Endeavour 463 & May 2009 & $5^{\circ} \mathrm{S}-3^{\circ} \mathrm{N}, 23^{\circ} \mathrm{W}$ & 800 \\
\hline Meteor 80/1 & October-November 2009 & $6^{\circ} \mathrm{S}-15^{\circ} \mathrm{N}, 23^{\circ} \mathrm{W}$ & 4500 \\
\hline Meteor 80/1 & November 2009 & $6^{\circ} \mathrm{S}-15^{\circ} \mathrm{N}, 23^{\circ} \mathrm{W}$ & 600 \\
\hline Meteor 81/1 & February 2010 & $11.5^{\circ} \mathrm{S}-13^{\circ} \mathrm{N}, 22^{\circ} \mathrm{W}$ & 1200 \\
\hline Maria S. Merian $18 / 2$ & May 2011 & $0^{\circ}-15^{\circ} \mathrm{N}, 23^{\circ} \mathrm{W}$ & 1600 \\
\hline Maria S. Merian $18 / 2$ & May-June 2011 & $5^{\circ} \mathrm{S}-5^{\circ} \mathrm{N}, 23^{\circ} \mathrm{W}$ & 5200 \\
\hline Maria S. Merian $18 / 3$ & June 2011 & $4^{\circ}-14^{\circ} \mathrm{N}, 23^{\circ} \mathrm{W}$ & 1320 \\
\hline Ron Brown 11/01 & August 2011 & $0^{\circ}-20.5^{\circ} \mathrm{N}, 23^{\circ} \mathrm{W}$ & 700 \\
\hline Maria S. Merian 22 & October-November 2012 & $6^{\circ} \mathrm{S}-8^{\circ} \mathrm{N}, 23^{\circ} \mathrm{W}$ & 5200 \\
\hline Maria S. Merian 22 & November 2012 & $0^{\circ}-15^{\circ} \mathrm{N}, 23^{\circ} \mathrm{W}$ & 1400 \\
\hline
\end{tabular}

by Schopf et al. [1981, their Eq. (2.2)] without making the quasigeostrophic approximation to study the beta dispersion of low-frequency Rossby waves. A ray path $\mathbf{x}(t)$ is obtained by integrating the local group velocity:

$$
\frac{d \mathbf{x}}{d t}=\mathbf{c}_{g}=\left(\frac{\partial \omega}{\partial k}, \frac{\partial \omega}{\partial l}\right)
$$

The change of local wavenumber and frequency along the ray path are given by (see Gill 1982, chapter 8.12.3)

$$
\begin{aligned}
& \frac{\mathrm{D}_{r} k}{\mathrm{D} t}=-\sum_{i} \frac{\partial \omega}{\partial \mu_{i}} \frac{\partial \mu_{i}}{\partial x}, \\
& \frac{\mathrm{D}_{r} l}{\mathrm{D} t}=-\sum_{i} \frac{\partial \omega}{\partial \mu_{i}} \frac{\partial \mu_{i}}{\partial y}, \quad \text { and } \\
& \frac{\mathrm{D}_{r} \omega}{\mathrm{D} t}=\sum_{i} \frac{\partial \omega}{\partial \mu_{i}} \frac{\partial \mu_{i}}{\partial t}=0,
\end{aligned}
$$

where $\mathrm{D}_{r} / \mathrm{D} t=(\partial / \partial t)+\mathbf{c}_{g} \cdot \nabla$ and $\mu=\left(U, V, \tilde{f}_{x}, \tilde{f}_{y}, R_{c}\right)$ is the set of spatially varying parameters of the local dispersion relation Eq. (11). The spatial derivatives of $\mu$ are evaluated with centered differences in spherical coordinates on the same grid as the shallow-water model and subsequently are bilinearly interpolated onto the location of the ray path to perform the Lagrangian integration of Eqs. (13) and (14). The rays are initialized at a distance of $2^{\circ}$ off the eastern boundary with frequency $\omega=\omega_{f}$, meridional wavenumber $l=0$, and the zonal wavenumber is chosen to satisfy the local dispersion relation Eq. (11).

\section{c. Barotropic mean flow}

Both the shallow-water model and the ray-tracing model require an estimate of the barotropic mean flow. In this paper, the mean flow felt by the Atlantic EDJs is estimated on the basis of zonal velocity observations collected from 22 ship sections at $23^{\circ} \mathrm{W}$ (listed in Table 1 ). The maximum measuring depth and temporal distribution of these sections is shown in Fig. 1. Each section consists of shipboard ADCP and lowered ADCP measurements, which are merged and interpolated according to Brandt et al. (2010) to a grid reaching to $5^{\circ} \mathrm{N} / \mathrm{S}$ and down to 5000-m depth with a horizontal resolution of $0.05^{\circ}$ and a vertical resolution of $10 \mathrm{~m}$. There is no extrapolation performed, hence the grid points below the maximum measuring depth of the individual section or outside its lateral bounds are considered as missing values. At each grid point the available data are then averaged to give a composite section shown in the left panel of Fig. 2. The composite section nicely reproduces the banded zonal velocity structure of the Atlantic Equatorial Intermediate Current System with the eastwarddirected NICC and SICC at $2^{\circ} \mathrm{N} / \mathrm{S}$ and westward velocity at about $3.5^{\circ}-4^{\circ} \mathrm{N} / \mathrm{S}$. These bands almost reach down to the seafloor and increase in amplitude toward the surface. On the equator the composite still shows remnants of the EDJs with maximum velocities of $10 \mathrm{~cm} \mathrm{~s}^{-1}$ at $400-\mathrm{m}$ depth. 


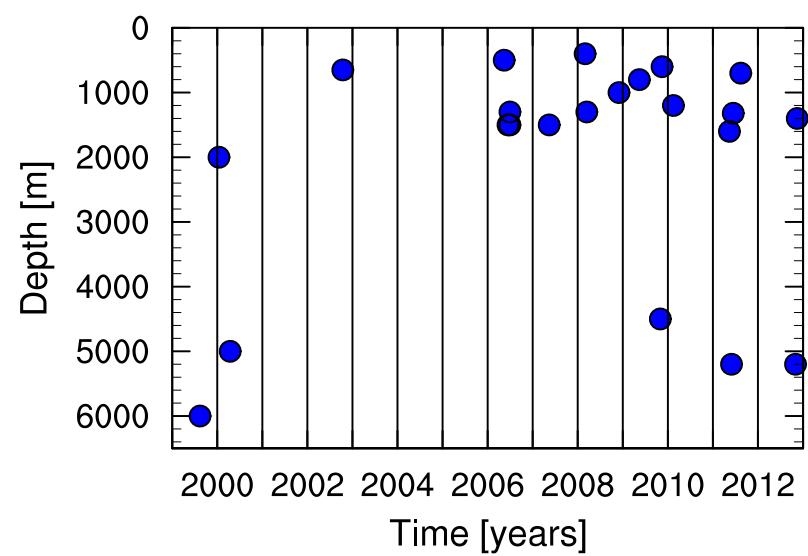

FIG. 1. Maximum measurement depth and temporal distribution of the cruises listed in Table 1.

To compute the barotropic mean flow, the composite section is vertically averaged between $300-$ and $5000-\mathrm{m}$ depth (or the bottom if shallower). The upper $300 \mathrm{~m}$ of the water column are excluded to remove the otherwise dominating signal of near-surface and thermocline currents, particularly the Equatorial Undercurrent. Please note that the order of the averaging, first with respect to the ship sections and then with respect to depth, gives more weight to the relatively sparse observations at greater depth (only five sections reaching below $2000 \mathrm{~m}$; see Fig. 1) and hence prevents a bias of the barotropic flow toward higher velocities due to a generally surface intensified zonal velocity structure. The barotropic mean flow profile computed from the composite zonal velocity section is shown in the right panel of Fig. 2 together with the depth-averaged flow of each individual section excluding depths with missing data. The barotropic mean flow again exhibits the series of alternating current bands being almost symmetric about the equator. Along the equator and at $3^{\circ} \mathrm{N} / \mathrm{S}$ the barotropic zonal velocity is westward with a magnitude of about $2 \mathrm{~cm} \mathrm{~s}^{-1}$. The NICC and SICC at $2^{\circ} \mathrm{N} / \mathrm{S}$ show up with velocities exceeding $4 \mathrm{~cm} \mathrm{~s}^{-1}$. Especially north of the equator, the location of maximum depth-averaged zonal velocity exhibits some variability among the individual sections; however, the banded structure of the zonal flow qualitatively remains the same among almost all sections.

To guarantee a divergence-free barotropic velocity field for the simulations with the shallow-water model and the ray-tracing model, we use a streamfunction of the velocity as input for the models rather than the velocity itself. This streamfunction is computed as the product of a single streamfunction profile at $23^{\circ} \mathrm{W}$ and an assumed zonal structure. The streamfunction profile at $23^{\circ} \mathrm{W}$ is obtained by integrating the zonal barotropic velocity profile, derived from the ship sections, in the meridional direction. Subsequently, the streamfunction profile is interpolated to the meridional axis of the model domain using spline interpolation with two additional supporting points added at the model boundaries to force the streamfunction to reach zero there. Since the resulting streamfunction profile has identical value at both meridional boundaries, there is no net zonal transport. The inevitable, compensating zonal flow is balanced between the hemispheres to some extent by removing the mean of the streamfunction profile before the interpolation was performed. Finally, the streamfunction profile was smoothed with a running mean filter of window size $0.5^{\circ}$ to reduce the noise in its second derivative that would otherwise lead to small-scale barotropic instability of the associated mean flow. However, this correction does not strongly affect the mean flow itself as one can see in the right panel of Fig. 2, where both the zonal flow from observations and the flow derived from the processed streamfunction profile are shown. These two profiles are almost indistinguishable. The assumed zonal structure function $f_{\lambda}$ is a linear combination of two hyperbolic tangents to form a plateau, that is,

$$
\begin{aligned}
f_{\lambda}= & \frac{1}{2}\left\{\tanh \left[\frac{1}{L_{c}}\left(\lambda-\lambda_{W}-\lambda_{0}\right)\right]\right. \\
& \left.-\tanh \left[\frac{1}{L_{c}}\left(\lambda-\lambda_{E}+\lambda_{0}\right)\right]\right\},
\end{aligned}
$$

where $L_{c}$ corresponds to the length scale of the boundary layer and is set to $1^{\circ}, \lambda_{W}$ and $\lambda_{E}$ are the coordinates of the western and eastern boundary, respectively, and $\lambda_{0}=5^{\circ}$ sets the distance of the boundary layer's center to the boundary. The streamfunction resulting from the product of the streamfunction profile and the zonal structure function is shown in the right panel of Fig. 3.

\section{Results}

In this section, we present the results from three classes of experiments that are all repeated identically with and without a mean flow, denoted as noMF and obsMF, respectively: (section 3a) ray tracing of free inviscid, long Rossby waves radiated at the eastern boundary to investigate the impact of the mean flow on the inviscid basin mode; (section 3b) the sensitivity of the resonance behavior of the shallow-water model along the equator to the gravity wave speed of the chosen vertical normal mode; and (section 3c) the impact of the mean flow on the structure of the equatorial basin mode in the presence of lateral mixing of momentum.

\section{a. Influence of the mean flow on the inviscid-free basin mode}

When the equatorial Kelvin wave impinges on the eastern boundary, it generates a coastal Kelvin wave, 

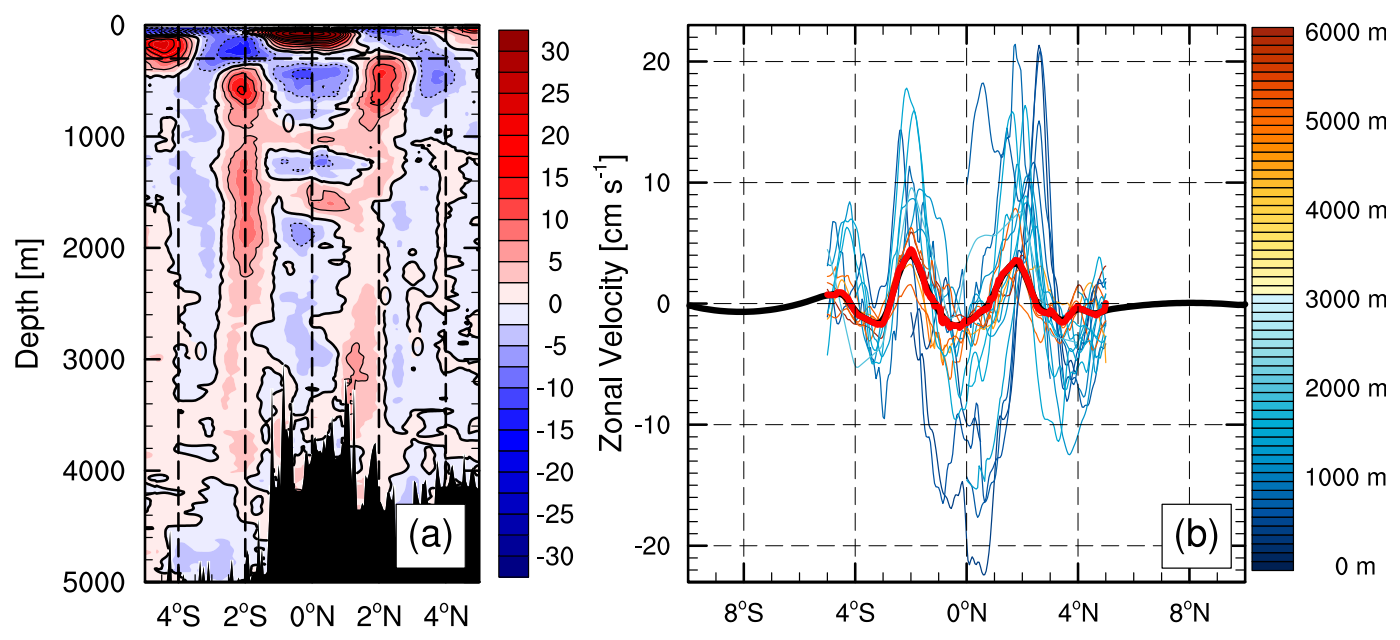

FIG. 2. (a) Composite section of zonal velocity along $23^{\circ} \mathrm{W}$ derived from the cruises listed in Table 1 . Negative values indicate westward flow and are shown using dotted lines. The contour interval is $5 \mathrm{~cm} \mathrm{~s}^{-1}$. Only data below the horizontal dashed line at 300-m depth are used to compute the mean zonal flow. (b) Barotropic zonal mean flow through $23^{\circ} \mathrm{W}$ derived directly by averaging from (a) and from the streamfunction used as model input (thick red and black line, respectively). Note that both lines are almost indistinguishable. The thin colored lines correspond to the depth-averaged flow derived from each individual cruise, where the averaging is performed solely over the available data of the particular cruise. The color of the line corresponds to the respective depth to which measurements were made, as indicated by the colorbar on the right.

cycling along the boundaries of the domain, a long Rossby wave traveling westward along the equator, and extratropical Rossby waves that propagate away from the eastern boundary. We initialize the ray-tracing model as described in section $2 b$, and the corresponding rays are shown in Fig. 3 for both configurations, with and without mean flow. For the case without mean flow, denoted as noMF, all rays are bent toward the equator due to beta dispersion (Schopf et al. 1981) and focus in the center of the domain. It is this caustic that leads to the singularity in the meridionally unbounded inviscid analytical solution of Cane and Moore (1981). If the mean flow is considered, the picture changes drastically. All rays shown initially turn away from the equator and continue to closely follow streamlines of the mean flow. Hence, the Rossby waves' local group velocity is rather determined by the mean flow itself than by the influence of the mean flow on the gradient of $\tilde{f}$. We confirmed this conclusion by an additional experiment (not shown), where $\tilde{f}$ is replaced by $f$ in Eq. (11), which leads to almost identical results. In addition, it is clear that the presence of the mean flow shields the equator from the extratropical Rossby waves. In particular, there is no focusing of energy on the equator, as in the noMF case.

\section{b. Resonance of a forced, weakly damped basin mode}

As mentioned earlier, the shallow-water model is driven by an oscillating zonal stress given by Eq. (6) to excite an equatorial basin mode. To test if the model resonates at the expected basin mode period a sensitivity experiment is done varying both the gravity wave speed and the forcing frequency $\omega_{f}$, but keeping the amplitude of the forcing fixed. Here, the spatial pattern of the forcing $X_{0}$ corresponds to the case denoted as "equator" in section 2, and the applied level of eddy viscosity is $A=$ $10 \mathrm{~m}^{2} \mathrm{~s}^{-1}$, which is the lowest value we use for any of the experiments conducted with the shallow-water model. Assuming the mean flow does not vary with latitude over the width of the basin mode, the Doppler-shifted period $T$ of the basin mode set up by a Kelvin wave and the gravest long equatorial Rossby wave is given by

$$
T=\int_{\lambda_{W}}^{\lambda_{E}} \frac{a}{c+u_{\mathrm{eq}}}+\frac{a}{(c / 3)-u_{\mathrm{eq}}} d \lambda,
$$

where $c$ is the gravity wave speed, and $u_{\mathrm{eq}}$ is the zonal velocity of the mean flow along the equator. Figure 4 shows the amplitude of the basin mode measured as the root-mean-square $\left(\sqrt{\overline{u^{2}}}\right)$ of the model zonal velocity along the equator. The averaging is carried out from $30^{\circ}$ to $15^{\circ} \mathrm{W}$ and over one forcing period at the end of the model run, when the model is in a steady oscillating state. The boundary regions are excluded to remove any signal that arises from nonequatorial waves like coastal Kelvin waves. Also shown is the expected resonance period given by Eq. (17), and it is obvious that this simple relation fits well to the pronounced resonance of the model. A qualitative interpretation of the results from the ray-tracing model suggests a reduced resonance amplitude of the basin mode in the presence of the mean flow due to the 

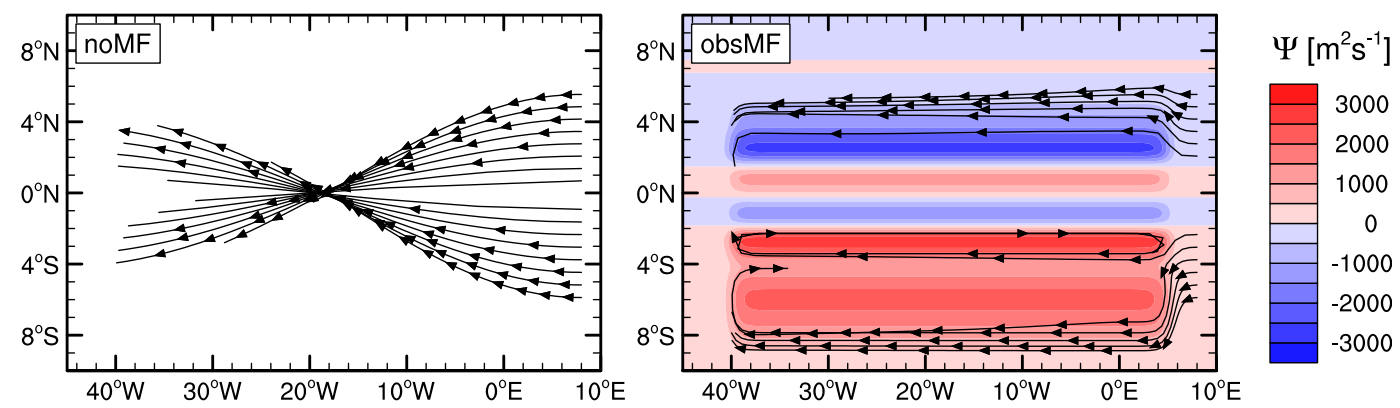

FIG. 3. Shading shows the streamfunction $\Psi$ used as model input for simulations without (noMF) and with (obsMF) mean flow. Note that for noMF the streamfunction is identically zero and therefore not shown. The black lines are the rays of free inviscid, long Rossby waves radiating in from the eastern boundary. The arrow heads are placed equidistant in time along the ray with a distance of approximately $3.1 \mathrm{yr}$. All rays are terminated if they reach $40^{\circ} \mathrm{W}$. Additionally, and only for obsMF, rays that get closer than $2^{\circ}$ to the equator are discontinued.

lack of energy focusing in the center of the basin. Indeed the resonance amplitudes of the case with mean flow are reduced by $24 \%-35 \%$ compared to the case without mean flow, although the amplitude of the forcing remains the same. Since the applied forcing is confined to the equator, this is further evidence for a shielding of the equatorial waveguide by the mean flow from off-equatorial Rossby waves.

\section{c. Impact of lateral mixing on a forced basin mode}

To elucidate both the influence of lateral mixing and the mean flow on the spatial structure of an equatorial basin mode, we conducted a set of experiments using the shallow-water model with and without considering a mean flow. The applied forcing pattern is again the one denoted as "equator", and the forcing period is set to 1670 days, which corresponds to the period of the EDJs observed in the Atlantic Ocean (Brandt et al. 2011). The gravity wave speed is set such that the amplitude of the model response along the equator is at the maximum for the case shown in Fig. 4 (parameters correspond to the location of the stars in Fig. 4). The results for two levels of eddy viscosity, 10 and $300 \mathrm{~m}^{2} \mathrm{~s}^{-1}$, are presented in Figs. 5 and 6, respectively, in terms of the amplitude and phase of a harmonic fitted to the time series of zonal velocity at each grid point. We consider only the last forcing period of the model run for the fit, when the model is in a steady oscillating state over large parts of the domain. Regions not plotted are where the adjustment to a steady oscillating state is not complete, and hence the fit explains less than $95 \%$ of the variance.

We first want to concentrate on the cases with relatively little mixing, that is, $A=10 \mathrm{~m}^{2} \mathrm{~s}^{-1}$. The amplitude of zonal velocity for the case without mean flow, depicted in Fig. 5a, is largest in the center of the domain where, in the inviscid case, the rays of the Rossby waves are focusing. This focusing is reflected in the weakly damped case by a widening of the meridional structure of amplitude about the equator to the east and to the west of the basin center. The respective phase for this case, shown in Fig. 5b, reveals a westward and, off the equator, also poleward phase propagation throughout the basin. Along the equator and east of $10^{\circ} \mathrm{W}$, the phase is almost constant while west thereof a constant zonal gradient can be seen. This gradient corresponds to a zonal-phase velocity of $5.5 \mathrm{~cm} \mathrm{~s}^{-1}$, which is in good agreement with the phase velocity of the gravest long equatorial Rossby wave of $5.7 \mathrm{~cm} \mathrm{~s}^{-1}$. For
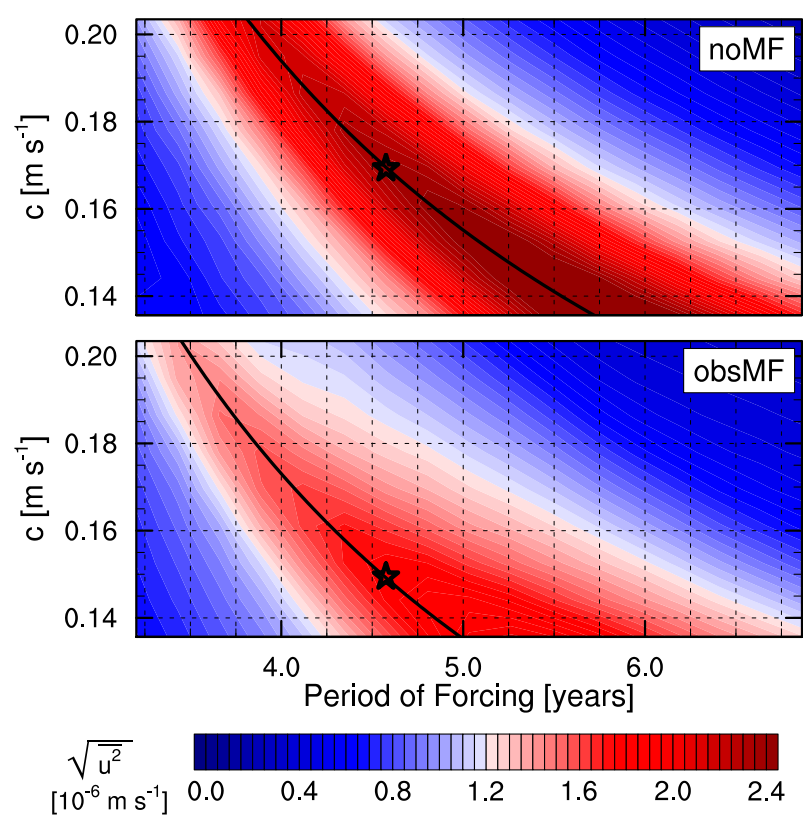

FIG. 4. Shading shows the root-mean-square of zonal velocity along the equator depending on the gravity wave speed $c$ and the forcing period for model configurations (top) without and (bottom) with mean flow. The averaging is carried out over one forcing period and from $30^{\circ}$ to $15^{\circ} \mathrm{W}$. The black line is the expected period for the gravest basin mode given by Eq. (17). The stars mark the parameters used for all other experiments in this paper. 

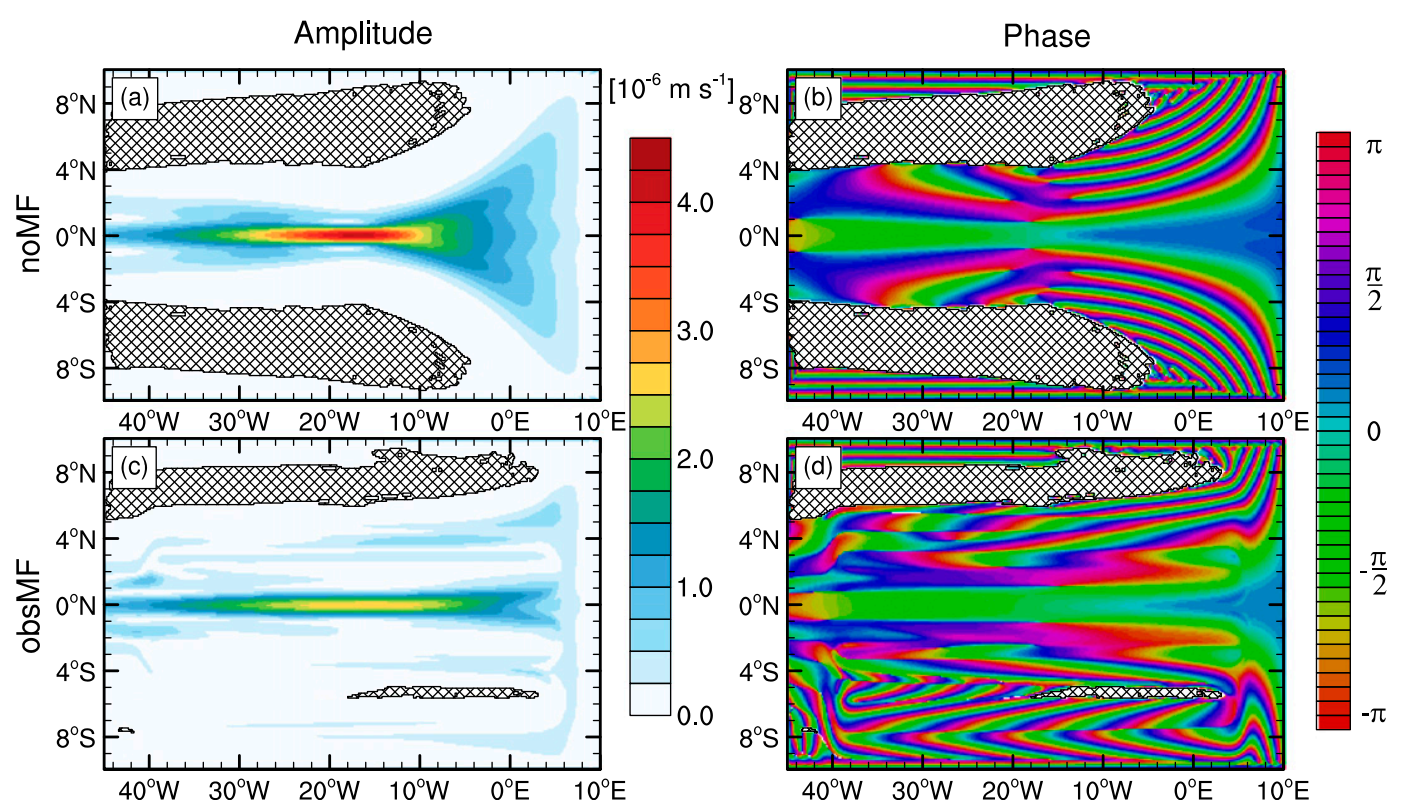

FIG. 5. (left) Amplitude ( $\left.\mathrm{m} \mathrm{s}^{-1}\right)$ and (right) phase of zonal velocity from experiments with little eddy viscosity $(A=$ $10 \mathrm{~m}^{2} \mathrm{~s}^{-1}$ ) applied for cases with (obsMF) and without (noMF) mean flow. Phase and amplitude are obtained by fitting a harmonic to the data at each location. Regions where the fit explains less than $95 \%$ of variance are masked cross hatched.

the case including the mean flow, the spatial structure of the amplitude, shown in Fig. 5c, is significantly different. The pronounced focusing in the eastern part of the basin has completely vanished and the maximum amplitude is about $30 \%$ smaller compared to the case without mean flow. However, the amplitude along the equator is not reduced homogeneously, but rather the maximum in the center of the domain is reduced. Generally, the amplitude structure is much more zonal and more confined to the equator compared to the case without mean flow. Off the equator a rich zonal structure has developed coinciding with bands of westward mean flow and with the ray paths of the inviscid Rossby waves depicted in Fig. 3 (obsMF). The corresponding phase is shown in Fig. $5 \mathrm{~d}$ and reflects the complex meridional structure by sharply separated zonal bands. Still, the phase propagation is westward throughout the basin, except for a small region between $4^{\circ}$ and $6^{\circ} \mathrm{S}$ where the phase propagation is reversed. There, the mean flow is eastward and hence carries the Rossby waves with it. On the equator, there is again a region of almost constant phase; however, it is smaller compared to noMF and extends from the eastern boundary to only $0^{\circ} \mathrm{E}$. West thereof, the zonal gradient of phase is again constant and the phase shift between the eastern and western boundary is almost the same as for the case without mean flow. The corresponding phase velocity is $6.9 \mathrm{~cm} \mathrm{~s}^{-1}$, which is in good agreement with the Doppler-shifted phase velocity of the gravest long equatorial Rossby wave of $7.1 \mathrm{~cm} \mathrm{~s}^{-1}$. Comparing both cases, it is apparent that the mean flow, just as in in the inviscid case, shields the equator from the off-equatorial Rossby waves, while for the case without mean flow there is a pronounced focusing in the center of the domain and generally a good agreement with the inviscid analytical solution of Cane and Moore (1981).

Now we increase the eddy viscosity to a moderate level of $A=300 \mathrm{~m}^{2} \mathrm{~s}^{-1}$. The amplitude for the cases without and with mean flow is shown in Figs. 6a and 6c, respectively. Interestingly, both have a remarkably similar structure: the amplitude is much reduced and its crossequatorial structure is wider compared to Figs. $5 \mathrm{a}$ and $5 \mathrm{c}$ due to the stronger lateral mixing of momentum, while the location of maximum amplitude is shifted eastward away from the center. For the case without mean flow, the signature of the off-equatorial maxima of zonal velocity associated with the gravest equatorial long Rossby wave is clearly visible at $2^{\circ} \mathrm{N} / \mathrm{S}$ almost throughout the domain. The corresponding phase, shown in Fig. 6b, supports this impression with a phase shift of $\pi$ between the equator and $2^{\circ} \mathrm{N} / \mathrm{S}$ away from the eastern boundary. Additionally, the off-equatorial wave fronts extend over the whole domain and propagate slowly poleward without any hint of focusing. The phase along the equator is of similar structure as the weakly damped case including mean flow; however, the gradient of phase west of $0^{\circ}$ is weaker, and the corresponding zonal-phase velocity is $10.1 \mathrm{~cm} \mathrm{~s}^{-1}$, almost twice the phase velocity of the inviscid gravest long Rossby wave. The amplitude of the case with mean flow (Fig. 6c) still shows the advection of Rossby waves 

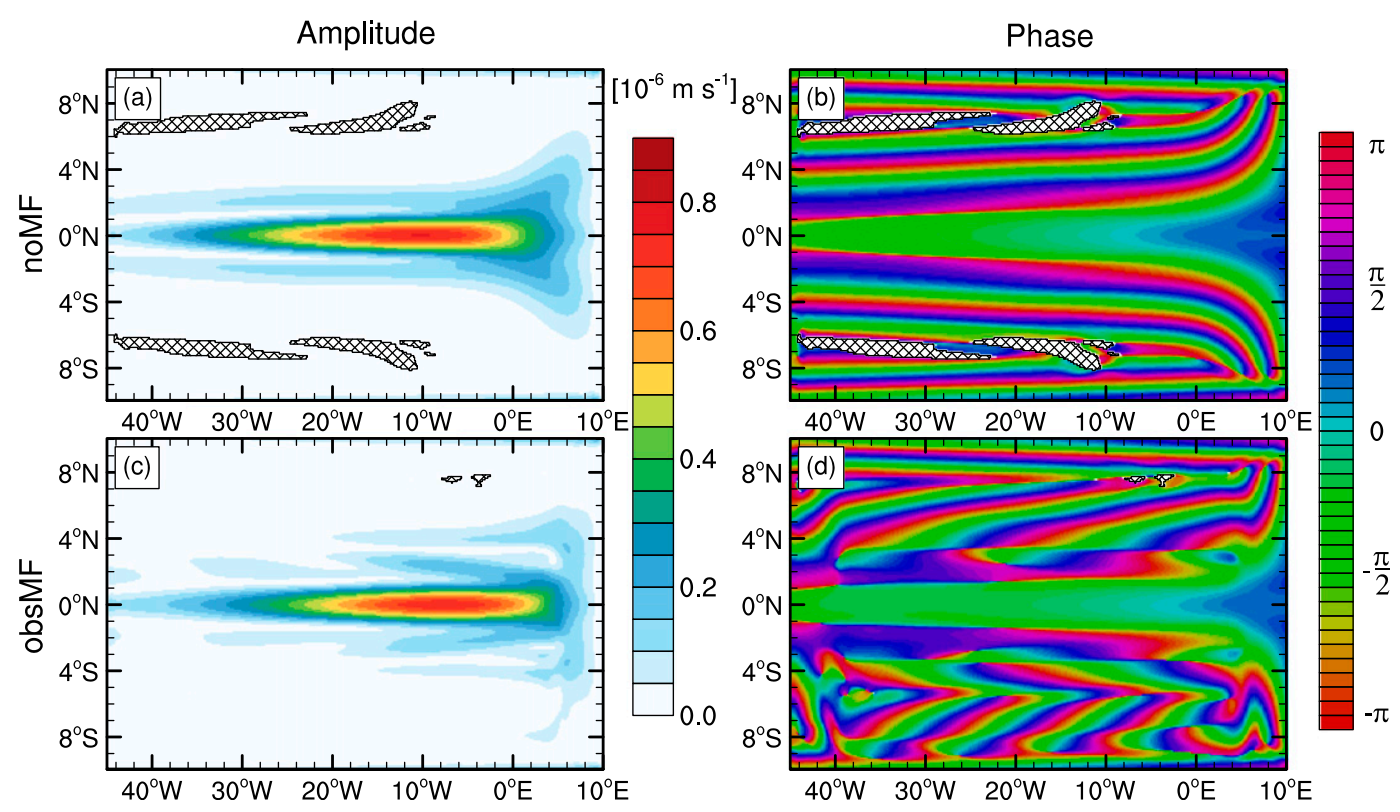

FIG. 6. As in Fig. 5, but for $A=300 \mathrm{~m}^{2} \mathrm{~s}^{-1}$. Note the different scale of the amplitude as compared to Fig. 5 .

from the eastern boundary along westward flow bands at $4^{\circ} \mathrm{N} / \mathrm{S}$ like in the respective weakly damped experiment; however, the structure is much coarser or blurred due to the increased lateral mixing. The phase propagation along the equator is also similar to the weakly damped obsMF case, except that the implied phase velocity west of $0^{\circ}$ is $14.8 \mathrm{~cm} \mathrm{~s}^{-1}$, which is again an increase of almost a factor of 2 compared to the weakly damped case. On the basis of the similarity of both wave fields in the vicinity of the equator and the missing focusing in the case without mean flow we conclude that both mean flow and the application of moderate eddy viscosity individually prevent the focusing of long Rossby waves due to beta dispersion. Additionally, the application of eddy viscosity significantly increases the westward-phase propagation along the equator for both cases noMF and obsMF.

As already mentioned, one striking feature of the Atlantic EDJs is their cross-equatorial width. Johnson and Zhang (2003) noted the dominance of the gravest Rossby wave on the structure of the EDJs and found that the EDJ's cross-equatorial width is about $50 \%$ larger than that of the gravest inviscid Rossby wave that has a vertical scale that best fits the observations. One can already see from a comparison of Figs. 5 and 6 that the crossequatorial width is sensitive to the applied rate of mixing. To get a more quantitative perspective, we conducted a sensitivity experiment with the shallow-water model, again with and without considering the mean flow. The forcing pattern is the one denoted as "equator", as before, the frequency and gravity wave speed corresponds to the previous set of experiments, and the lateral eddy viscosity varies from 10 to $600 \mathrm{~m}^{2} \mathrm{~s}^{-1}$. A bell function was fitted to the meridional profiles of the root-mean-square of zonal velocity at each line of longitude. Here, the averaging is done over a full basin mode cycle at the end of each run, when the model is in a steady oscillating state inside the equatorial band. The fitted function is proportional to $\exp \left(-\theta^{2} / L_{e}^{2}\right)$, where $L_{e}$ is the $e$-folding scale and will be taken as a synonym for the width. Following the scaling argument of Greatbatch et al. (2012), we expect a width of $L_{e}=\sqrt{2 / 3} R_{E} \approx 0.8 R_{E}$ in the inviscid limit. Figure 7 shows the dependence of $L_{e}$, normalized by the respective inviscid width, on both location and applied eddy viscosity. If no mean flow is taken into account (noMF), there is a pronounced tightening of the jet in the center of the basin for low levels of eddy viscosity and a widening to the east and to the west. This is again the signature of the Rossby wave focusing evident from Fig. 5a. However, reaching a level of $50 \mathrm{~m}^{2} \mathrm{~s}^{-1}$ for the eddy viscosity, the focusing is totally gone. Increasing the lateral mixing of momentum even further, the jet continues to broaden especially in the eastern part of the basin, and a positive zonal gradient of width establishes throughout the basin. The observed broadening of $50 \%$ compared to the expected inviscid width is reached for applied eddy viscosities of $100 \mathrm{~m}^{2} \mathrm{~s}^{-1}$ at $10^{\circ} \mathrm{W}$ and $400 \mathrm{~m}^{2} \mathrm{~s}^{-1}$ at $30^{\circ} \mathrm{W}$.

For the model linearized about the mean flow (obsMF), the picture changes significantly. There is no pronounced focusing for low levels of applied mixing; instead, the width appears to be fairly zonally uniform and close to the expected inviscid width. The range of eddy viscosity for which the cross-equatorial width shows 


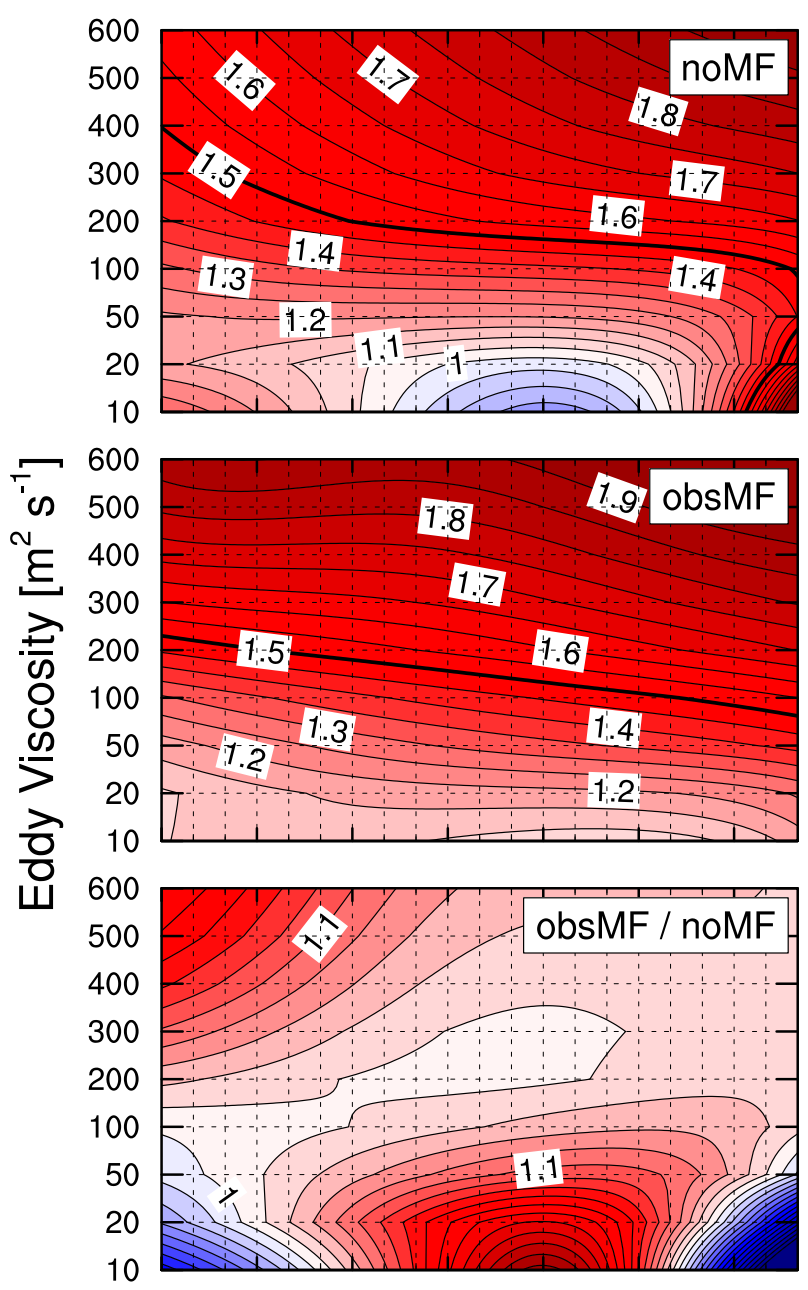

$30^{\circ} \mathrm{W} 27^{\circ} \mathrm{W} 24^{\circ} \mathrm{W} 21^{\circ} \mathrm{W} 18^{\circ} \mathrm{W} 15^{\circ} \mathrm{W} 12^{\circ} \mathrm{W}$

FIG. 7. (top) Shading and contour lines show the local width of the zonal jets along the equator, normalized by the width of the inviscid, gravest, long Rossby wave $(\sqrt{2 c / 3 \beta})$ for experiments without mean flow (noMF) and driven by the forcing denoted as "equator." The width is estimated as the $e$-folding scale of a Gaussian that is fitted to the meridional profile of the root-mean-square of zonal velocity, where the averaging is done over one forcing period. The contour interval is 0.05 and the 1.5 contour line is drawn thicker for convenience. (middle) Same as the top panel but with mean flow applied (obsMF). (bottom) Ratio of the upper two panels (obsMF/noMF). Values above one correspond to a wider relative cross-equatorial structure in the presence of mean flow compared to experiments without mean flow. The contour interval is 0.02 and the color scale differs from the upper two panels.

the observed broadening is between 75 to $220 \mathrm{~m}^{2} \mathrm{~s}^{-1}$ and thus lower than for the case without mean flow. The strong reduction of the upper range compared to noMF is related to an enhanced broadening with increasing eddy viscosity in the western part of the domain for eddy viscosities above $150 \mathrm{~m}^{2} \mathrm{~s}^{-1}$. This leads to a less pronounced zonal gradient of width and even almost constant width west of $22^{\circ} \mathrm{W}$ for eddy viscosities above $400 \mathrm{~m}^{2} \mathrm{~s}^{-1}$. The ratio of the normalized width of both cases, shown in Fig. 7 (obsMF/noMF), reveals that, in general, adding mean flow to the model gives a greater width compared to a model without mean flow and prohibits focusing, which is further evidence for its shielding effect. A possible explanation for the enhanced width is the alteration of the ambient vorticity and hence its meridional gradient by the zonal mean flow. Assuming a solely latitudinally varying mean flow, this influence can be estimated using an effective $\beta$, where $\beta_{\text {eff }}=\beta-U_{y y}$, and hence the effective radius of deformation $R_{E_{\text {eff }}}$ is given by

$$
R_{E_{\mathrm{eff}}}=\sqrt{\beta / \beta_{\mathrm{eff}}} R_{E}
$$

Figure 8 shows $\sqrt{\beta / \beta_{\text {eff }}}$ as a function of latitude for a mean flow profile taken from the center of the basin and suggests a widening effect from the mean flow of the order of $5 \%-10 \%$ near the equator. However, this overestimates the widening of the jet at most locations and levels of mixing. Only in the center of the basin for low mixing rates, where a focusing is prohibited by the mean flow, and west of $24^{\circ} \mathrm{W}$ when eddy viscosities are above $300 \mathrm{~m}^{2} \mathrm{~s}^{-1}$, can such a widening be found. At most locations and for viscosities above $50 \mathrm{~m}^{2} \mathrm{~s}^{-1}$, the widening with respect to the case without mean flow is less than $5 \%$.

To gain a better understanding of the effects of the mean flow on the two wave components of the basin mode, that is, the equatorial Kelvin wave and the Rossby wave, a set of experiments are conducted, where both the lateral mixing of momentum and the spatial pattern of the forcing are varied. Since the basin mode in the model is subject to dissipation, a forcing in the eastern part of the basin will favor the Rossby wave, while forcing in the west will emphasize the Kelvin wave. Figure 9 shows the width, normalized by the expected inviscid width $\sqrt{2 / 3} R_{E}$, again measured as the $e$-folding scale of a bell function, which is fitted to a meridional profile of the rootmean-square of zonal velocity. The averaging is calculated again over one full basin mode cycle at the end of each run, when the model is in a steady oscillating state but now also from $30^{\circ}$ to $10^{\circ} \mathrm{W}$; thus, an additional zonal averaging is implied. In fact, this gives a better comparability to the widening quantified by Johnson and Zhang (2003), since their estimate is based on zonally averaged data from hydrographic stations scattered over the whole equatorial Atlantic. In our analysis, the boundary regions are excluded to avoid effects arising from coastal Kelvin waves. The results for the cases without mean flow are in good agreement with the ones from Greatbatch et al. (2012). In fact, the simulations are identical but the analysis is slightly different. Greatbatch et al. (2012) 


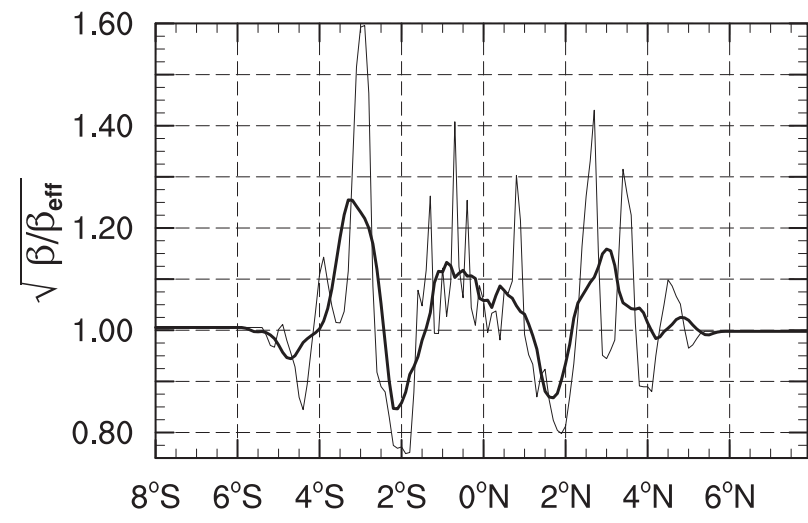

FIG. 8. Effect of the meridional curvature of the mean flow seen by the model on the equatorial radius of deformation as given by Eq. (18) (thin line). Values above one correspond to a larger effective equatorial radius of deformation compared to the case without mean flow. The mean flow profile is taken from the center of the basin, where the flow is purely zonal. Also shown is the running average with a window size of $1^{\circ}$ (thick line).

estimated the width by inferring the distance to the equator where the root-mean-square profile first drops below $e^{-1}$ times its value on the equator. However, since the profile nicely fits a bell function (not shown), both methods give almost identical results. Also shown is the dependence of width on the lateral mixing as obtained by the scale analysis of Greatbatch et al. (2012). The cases with the forcing applied in the western third of the basin exhibits the widest cross-equatorial structure for all levels of lateral mixing. The more the forcing region moves to the east, and hence the contribution of the Rossby wave increases, the narrower the jets are. This is not surprising since the Rossby wave does have a smaller width compared to the Kelvin wave. However, the sensitivity to the applied eddy viscosity appears to be weaker for the Rossby wave, that is, the cases where the forcing is applied more to the east. Adding the barotropic mean flow to the model increases the width for all forcing patterns at all levels of mixing, except for the case "east," that has the largest contribution of the Rossby wave to the basin mode. In this case, the width is increased for values of eddy viscosity below $200 \mathrm{~m}^{2} \mathrm{~s}^{-1}$ but decreases for values above when compared to noMF. The widening by the mean flow is largest if the forcing is confined to the western third of the domain with an increase of $10 \%-$ $20 \%$, which is roughly consistent with the estimate based on Eq. (18). Interestingly, the relative increase in width is of similar magnitude for the cases "center" and "full". Moreover, the steeper slopes of the lines corresponding to these three cases in Fig. 9 (obsMF), compared to (noMF), indicate a stronger sensitivity of width to the level of eddy viscosity in the presence of the mean flow. This cannot be seen for the forcing patterns "equator" and "east", which favor the Rossby wave, and suggests that in the presence of the barotropic mean flow, an increased momentum diffusion predominantly widens the Kelvin wave component of the equatorial basin mode.

\section{Summary and discussion}

In this work, we excited an equatorial basin mode in a shallow-water model for a single high-order baroclinic vertical normal mode, linearized about a barotropic mean flow, and took it as a simple model of the Atlantic equatorial deep jets (EDJs). The basin size and the forcing frequency correspond to the Atlantic Ocean, and the zonal mean flow is estimated from a collection of cruise data along $23^{\circ} \mathrm{W}$. This mean flow corresponds to the well-known equatorial Atlantic zonal current structure including the signature of the eastward-flowing North and South Intermediate Counter Currents (NICC and SICC, respectively) at $2^{\circ} \mathrm{N} / \mathrm{S}$ and of the westwarddirected currents at the equator [the Equatorial Intermediate Current (EIC)] and poleward of the NICC and SICC.

We have shown that the focusing of long equatorial Rossby waves due to beta dispersion, a prominent feature of the inviscid or weakly damped equatorial basin mode, is suppressed in the presence of the mean flow. This is due to the advection of the extratropical Rossby waves by the mean flow away from the equator and hence a leakage of energy to off-equatorial waveguides coinciding with the westward mean currents. However, applying sufficient lateral eddy viscosity to the model linearized about a state of rest also prohibits a focusing of the jets. We therefore conclude that a focusing of long Rossby waves along with a pronounced tightening of the jet in the center of the basin is not a likely feature of an equatorial basin mode in the real Atlantic Ocean and thus not of the EDJs.

The results also suggest that in the presence of the barotropic mean flow, an increased momentum diffusion predominantly widens the Kelvin wave component of the equatorial basin mode, while the Rossby wave appears to be confined by the flanking NICC and SICC. Indeed, the relation between the width of the basin mode and the strength of lateral eddy viscosity depends notably on the region where the forcing takes place: the farther to the east, the less sensitive is the width to the applied mixing. To broaden the zonal jets at the equator to a width of 1.5 times the expected inviscid width a lateral eddy viscosity of less than $200 \mathrm{~m}^{2} \mathrm{~s}^{-1}$ is required, regardless of whether the mean flow is considered or not. This threshold is below the estimate of $400 \mathrm{~m}^{2} \mathrm{~s}^{-1}$ by Brandt et al. (2008) for the equatorial Atlantic based on the oxygen budget along the equator. It 

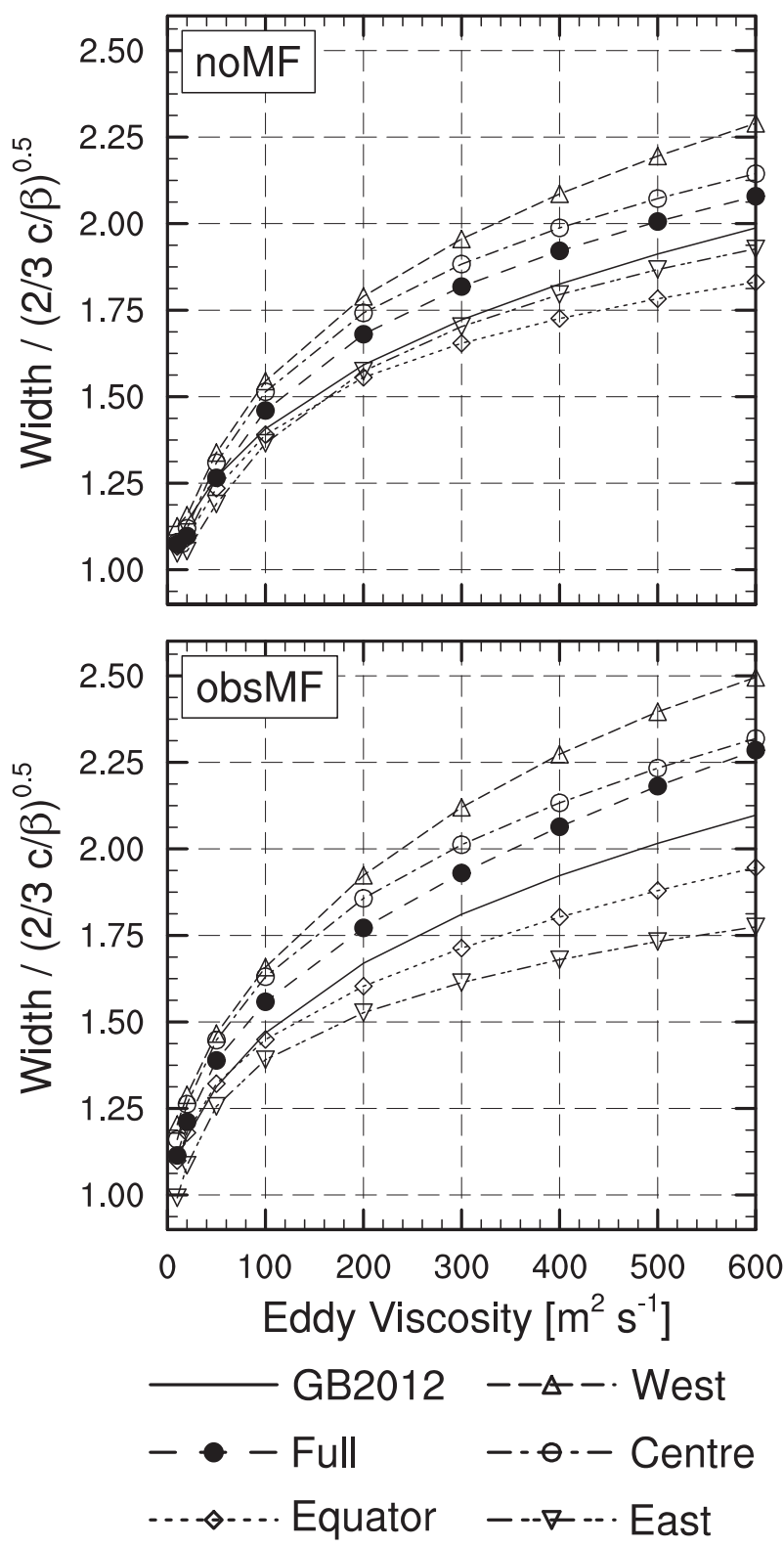

FIG. 9. Width of the modeled EDJs, normalized by the width of the inviscid, gravest, long Rossby wave for different forcing patterns and values of eddy viscosity. The width is estimated as the $e$-folding scale of a Gaussian fitted to the meridional profile of the root-meansquare of zonal velocity, where the average is taken from $30^{\circ}$ to $10^{\circ} \mathrm{W}$ and over one forcing period. The panel indicating noMF is without and obsMF is with mean flow. The solid line corresponds to results from the scaling analysis of Greatbatch et al. (2012) based on the model parameters for each of the two cases.

still remains an open question how these two processes, the effect of the mean flow and of lateral mixing of momentum, compare with the impact of nonlinear effects. Here, further research is needed; however, the model framework used in this work will not be applicable because the consideration of nonlinear terms will also introduce interactions between different vertical normal modes.

It should be noted here that we have not considered two effects in this study, which might affect the results and could be tested with the same model and methodology: (i) the shape of the equatorial Atlantic basin and (ii) the zonal structure of the mean flow. The work of Cane and Gent (1984) suggests that the slope of the South American coast might reduce the amplitude of the reflected equatorial Kelvin wave and therefore of the basin mode but would not otherwise change the results. The east-west-directed coast of the Gulf of Guinea at $5^{\circ} \mathrm{N}$, on the other hand, is distant enough from the equator to not directly influence the equatorial waves for a high vertical mode such as considered here. It will, however, affect the focusing of extratropical Rossby waves of the basin mode. These waves will not focus in the center of the basin even in the case of no mean flow and no lateral mixing. More seriously, the zonal structure of the barotropic mean flow that is assumed in this work is highly simplified. In observations, the NICC and SICC can only be found west of $0^{\circ}$ (Bourlès et al. 2003) and the lack of zonal flow in the eastern part of the domain might influence both the propagation of the extratropical Rossby waves and the equatorial confinement of the equatorial Rossby waves. However, the similarity of the basin mode in both models (with and without mean flow) in the presence of lateral mixing (sufficient to explain the observed width of the EDJs; Fig. 6) suggests that the consideration of the coastline of the Gulf of Guinea and the zonal structure of the mean flow would not have a major impact on our results.

We want to point out that our model, a basin mode of a single baroclinic mode, is not able to capture vertical propagation, either of phase or energy, and hence lacks this important feature of the Atlantic EDJs. In a mode spectrum of moored zonal velocity measurements at $23^{\circ} \mathrm{W}$, presented by Brandt et al. (2008), the EDJs appear as a broad peak centered around mode number 15 , reaching from mode 10 to 20 . Since the gravity wave speeds for this range of modes are not substantially different, the broad resonance peak, shown in Fig. 4, suggests that a forcing of a single frequency is able to excite a set of quasi-resonant basin modes in a band of vertical normal modes. These would show up as vertically propagating stacked jets just like the observed EDJs. The direction of propagation would be determined only by the vertical structure of the forcing and hence how well it projects onto the individual mode structure. This idea could explain the dynamics of the EDJs solely on the basis of linear theory and will be the subject of further research. 
Acknowledgments. MC is grateful for support from the German Federal Ministry of Education and Research (BMBF) Miklip project through the MODINI project, and RJG is grateful for continuing support from GEOMAR Helmholtz Centre for Ocean Research Kiel. This study has been supported by the Deutsche Forschungsgemeinschaft as part of the Sonderforschungsbereich 754 "Climate Biogeochemistry in the Tropical Ocean." We are grateful to two anonymous reviewers for their constructive comments.

\section{REFERENCES}

Ascani, F., E. Firing, P. Dutrieux, J. P. McCreary, and A. Ishida, 2010: Deep equatorial ocean circulation induced by a forced-dissipated Yanai beam. J. Phys. Oceanogr., 40, 1118-1142, doi:10.1175/ 2010JPO4356.1.

Bourlès, B., and Coauthors, 2003: The deep currents in the eastern equatorial Atlantic Ocean. Geophys. Res. Lett., 30, 8002, doi:10.1029/2002GL015095.

Brandt, P., V. Hormann, B. Bourlès, J. Fischer, F. A. Schott, L. Stramma, and M. Dengler, 2008: Oxygen tongues and zonal currents in the equatorial Atlantic. J. Geophys. Res., 113, C04012, doi:10.1029/2007JC004435.

— - — A. Körtzinger, M. Visbeck, G. Krahmann, L. Stramma, R. Lumpkin, and C. Schmid, 2010: Changes in the ventilation of the oxygen minimum zone of the tropical North Atlantic. J. Phys. Oceanogr., 40, 1784-1801, doi:10.1175/2010JPO4301.1.

- , A. Funk, V. Hormann, M. Dengler, R. J. Greatbatch, and J. M. Toole, 2011: Interannual atmospheric variability forced by the deep equatorial Atlantic Ocean. Nature, 473, 497-500, doi:10.1038/nature10013.

—_ and Coauthors, 2012: Ventilation of the equatorial Atlantic by the equatorial deep jets. J. Geophys. Res., 117, C12015, doi:10.1029/2012JC008118.

Bunge, L., C. Provost, B. L. Hua, and A. Kartavtseff, 2008: Variability at intermediate depths at the equator in the Atlantic Ocean in 2000-06: Annual cycle, equatorial deep jets, and intraseasonal meridional velocity fluctuations. J. Phys. Oceanogr. 38, 1794-1806, doi:10.1175/2008JPO3781.1.

Cane, M. A., and D. W. Moore, 1981: A note on low-frequency equatorial basin modes. J. Phys. Oceanogr., 11, 1578-1584, doi:10.1175/ 1520-0485(1981)011<1578:ANOLFE $>2.0 . C O ; 2$.

— waves at arbitrary western boundaries. J. Mar. Res., 42, 487502, doi:10.1357/002224084788505988.
Chang, P., and S. Philander, 1989: Rossby wave packets in baroclinic mean currents. Deep-Sea Res., 36A, 17-37, doi:10.1016/ 0198-0149(89)90016-2.

d'Orgeville, M., B. L. Hua, and H. Sasaki, 2007: Equatorial deep jets triggered by a large vertical scale variability within the western boundary layer. J. Mar. Res., 65, 1-25, doi:10.1357/ 002224007780388720 .

Eriksen, C. C., 1982: Geostrophic equatorial deep jets. J. Mar. Res., 40, 143-157.

Firing, E., 1987: Deep zonal currents in the central equatorial Pacific. J. Mar. Res., 45, 791-812, doi:10.1357/002224087788327163.

Gill, A. E., 1982: Atmosphere-Ocean Dynamics. Academic Press, $664 \mathrm{pp}$.

Gouriou, Y., and Coauthors, 2001: Deep circulation in the equatorial Atlantic Ocean. Geophys. Res. Lett., 28, 819-822, doi:10.1029/ 2000GL012326.

Greatbatch, R. J., P. Brandt, M. Claus, S.-H. Didwischus, and Y. Fu, 2012: On the width of the equatorial deep jets. J. Phys. Oceanogr., 42, 1729-1740, doi:10.1175/JPO-D-11-0238.1.

Hayes, S. P., and H. B. Milburn, 1980: On the vertical structure of velocity in the eastern equatorial Pacific. J. Phys. Oceanogr., 10, 633-635, doi:10.1175/1520-0485(1980)010<0633: OTVSOV $>2.0 . \mathrm{CO} ; 2$.

Hua, B. L., D. W. Moore, and S. Le Gentile, 1997: Inertial nonlinear equilibration of equatorial flows. J. Fluid Mech., 331, 345-371, doi:10.1017/S0022112096004016.

Johnson, G. C., and D. Zhang, 2003: Structure of the Atlantic Ocean equatorial deep jets. J. Phys. Oceanogr., 33, 600-609, doi:10.1175/1520-0485(2003)033<0600:SOTAOE > 2.0.CO;2.

— E. Kunze, K. E. McTaggart, and D. W. Moore, 2002: Temporal and spatial structure of the equatorial deep jets in the Pacific Ocean. J. Phys. Oceanogr., 32, 3396-3407, doi:10.1175/ 1520-0485(2002)032<3396:TASSOT >2.0.CO;2.

Leetmaa, A., and P. F. Spain, 1981: Results from a velocity transect along the equator from 125 to $159^{\circ} \mathrm{W}$. J. Phys. Oceanogr., 11, 1030-1033, doi:10.1175/1520-0485(1981)011<1030: RFAVTA $>2.0 . \mathrm{CO} ; 2$

Luyten, J. R., and J. C. Swallow, 1976: Equatorial undercurrents. Deep-Sea Res. Oceanogr. Abstr., 23, 999-1001, doi:10.1016/ 0011-7471(76)90830-5.

Richardson, P. L., and D. M. Fratantoni, 1999: Float trajectories in the deep western boundary current and deep equatorial jets of the tropical Atlantic. Deep-Sea Res. II, 46, 305-333, doi:10.1016/S0967-0645(98)00100-3.

Sadourny, R., 1975: The dynamics of finite-difference models of the shallow-water equations. J. Atmos. Sci., 32, 680-689, doi:10.1175/1520-0469(1975)032<0680:TDOFDM >2.0.CO;2.

Schopf, P. S., D. L. T. Anderson, and R. Smith, 1981: Betadispersion of low-frequency Rossby waves. Dyn. Atmos. Oceans, 5, 187-214, doi:10.1016/0377-0265(81)90011-7. 